\title{
EDITOR'S PREFACE TO THE DIALOGUE SERIES
}

\section{THE DIALOGUE SERIES}

This dialogue is the first to appear in Macroeconomic Dynamics. Other dialogues on topics of importance to macroeconomics will appear occasionally in this journal. Unlike interviews appearing in the $M D$ Interview series, conversations appearing in the $M D$ Dialogue series will not be focused on the life and work of a participant but rather on a particular research topic. Another distinction between the two series is the general format and structure. Each interview in the $M D$ Interview series will include only one interviewee, although there may be multiple interviewers who jointly conduct an interview with a single interviewee (e.g., the interview of David Cass by Stephen Spear and Randall Wright in the preceding issue). In contrast, each dialogue will include at least two direct dialogue participants, and may or not be conducted by a third party to the conversation.

\section{THIS DIALOGUE}

Dialogues in the $M D$ Dialogue series often will be in the form of unstructured conversations among the participants, but these dialogues sometimes will be conducted more formally by an interviewer. This first dialogue is in the form of two separate conversations, both conducted by David Colander. One conversation is with James Tobin and the other is with Robert Shiller. The reason for this somewhat unusual, and rather unanticipated, format is explained by David Colander in his introduction. However the focus of these two conversations clearly distinguishes this exchange as a dialogue, rather than as two interviews, and hence is a suitable, as well as very interesting and enlightening, exchange with which to begin this $M D$ Dialogue series.

Readers interested in a traditional interview of James Tobin, focused on his life and works, may be interested to know that such an interview, conducted by Robert Shiller, is forthcoming in the Cambridge University Press journal, Econometric Theory.

\section{William A. Barnett}

